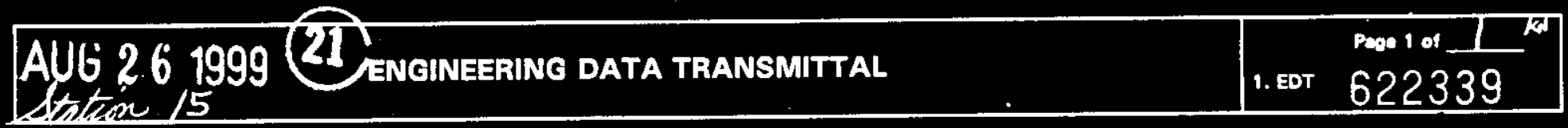

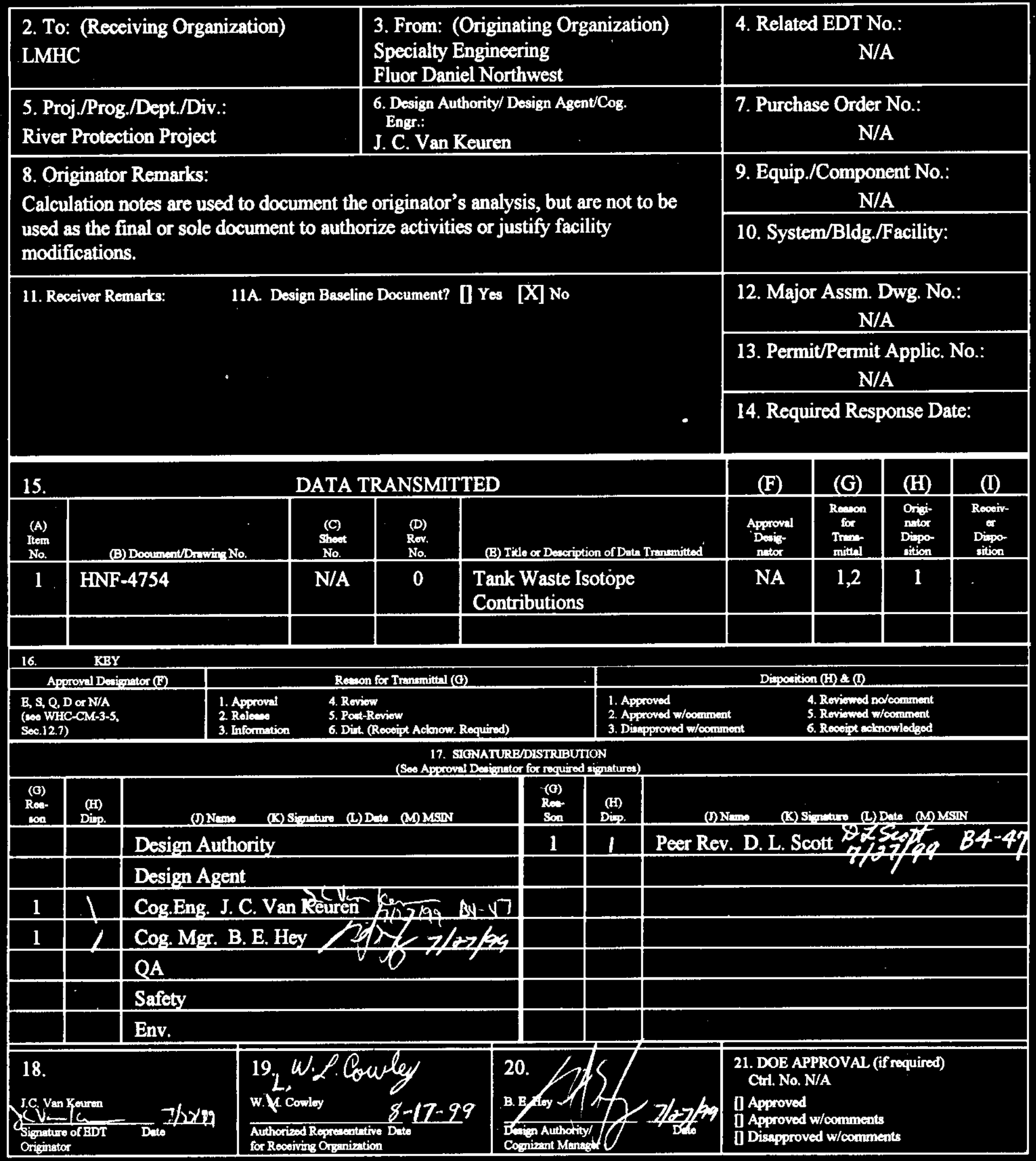




\title{
TANK WASTE ISOTOPE CONTRIBUTIONS
}

\author{
J. C. Van Keuren
}

Fluor Daniel Northwest Hanford, Richland, WA 99352

U.S. Department of Energy Contract DE-AC06-96RL13200

EDT/ECN: 622339

UC: 510

Org Code: 403

B\&R Code: EW3130010

Charge Code: $1.01952 / B B 20 \quad$ HN920471

Total Pages: 18

Key Words: Dose calculation, Dose conversion factors

Abstract: This document presents the results of a calculation to determine the relative contribution of selected isotopes to the inhalation and ingestion doses for a postulated release of Hanford tank waste. The fraction of the dose due to ${ }^{80} \mathrm{Sr},{ }^{80} \mathrm{Y},{ }^{137} \mathrm{Cs}$ and the alpha emitters for single shell solids and liquids, double shell solids and liquids, aging waste solids and liquids and all solids and liquids. An effective dose conversion factor was also calculated for the alpha emitters for each composite of the tank waste.

TRADEMARK DISCLAIMER. Reference hereln to any specilic commercial product, procees, or service by trade name, trademark, manufecturer, or otherwhe, does not necessarly constitute or imply its endorsement, recommendation, or favoring by the Unted States Government or any agency thereof or lis contractors or subcontractors.

Pinted In the United States of America. To obtein copies of this document, contact: Document Control Services, P.O. Box 9s0, Mallatop H8-08, Richland WA se352, Phone (509) 372-2420; Fax (509) 376-4989.
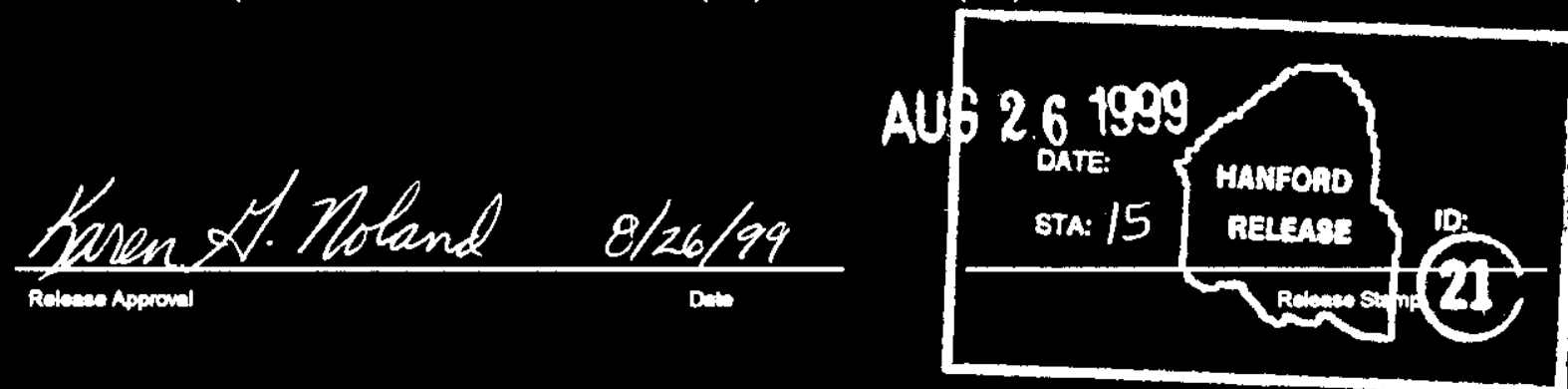

Approved for Public Release 


\subsection{Introduction}

The purpose of this report is document two calculations relative to the fractional contribution of different isotopes used for the Tank Waste Remediation Systems (TWRS) Basis for Interim Operation (BIO) and Final Safety Analysis Report (FSAR) dose calculations. The dose calculations are based on data given in SARR-016, Rev 2, (WHC-SD-WM-SARR-016, 1996). The first calculation determines the fraction of dose due to ${ }^{\circ} \mathrm{Sr},{ }^{90},{ }^{137} \mathrm{Cs}$, and the alpha emitters. Both inhalation and ingestion doses are considered. The second calculation looks at the average dose conversion factors for the alpha emitters. The results of the first calculation are given in Section 2 and the second in Section 3. Details of the calculations are given in Appendices A, B and C.

\subsection{Fraction of Dose Due to Selected Isotopes}

\subsection{Inhalation doses}

The inhalation unit liter doses (ULD) for each composite are calculated in WHC-SD-WMSARR-016, (1996) using the following formula:

$$
\mathrm{ULD}=\Sigma\left(D C F_{1} \times A_{1}\right)
$$

Where $D C F_{1}=$ Dose conversion factors for the ith isotope $A_{1}=$ Activity for the ith isotope

A calculation has been made to determine the fraction of the doses due to ${ }^{\infty} \mathrm{Sr}^{\infty}{ }^{\infty} \mathrm{Y}$, ${ }^{137} \mathrm{Cs}$, and the alpha emitters. The alpha emitters in the composites are ${ }^{237} \mathrm{~Np},{ }^{238} \mathrm{Pu}$, ${ }^{230} \mathrm{Pu},{ }^{241} \mathrm{Am}$, and ${ }^{24} \mathrm{Cm}$. Alpha emitters are grouped together since activities may be reported as "total alpha." The fraction of the ULD of the selected isotopes is determined by dividing the ULD calculated using only the 8 isotopes by the ULD calculated with all the isotopes. The inhalation dose conversion factors are taken from EPA, 1988 and are based on ICRP 26 and 30 methodology. The calculations are shown in Appendix $A$ and the results are summarized in Table 1.

Table 1. Fraction of ULD for Inhalation Dose for ${ }^{80} \mathrm{Sr},{ }^{80},{ }^{137} \mathrm{Cs}$, and the Alpha Emitters.

\begin{tabular}{|l|c|c|c|}
\hline Composite & $\begin{array}{c}\text { ULD for selected } \\
\text { isotopes (SV/L) }\end{array}$ & $\begin{array}{c}\text { ULD for all Isotopes } \\
\text { (SV/L) }\end{array}$ & $\begin{array}{c}\text { Fraction of } \\
\text { Contribution of } \\
\text { Selected lsotopes }\end{array}$ \\
\hline SST Liquids & $1.02 \mathrm{E}+04$ & $1.10 \mathrm{E}+04$ & 0.931 \\
\hline SST Solids & $2.13 \mathrm{E}+05$ & $2.21 \mathrm{E}+05$ & 0.965 \\
\hline DST Liquids & $6.01 \mathrm{E}+03$ & $6.06 \mathrm{E}+03$ & 0.993 \\
\hline DST solids & $5.20 \mathrm{E}+05$ & $5.29 \mathrm{E}+05$ & 0.984 \\
\hline AWF Liquids & $1.42 \mathrm{E}+03$ & $1.42 \mathrm{E}+03$ & 0.999 \\
\hline AWF Solids & $1.72 \mathrm{E}+03$ & $1.73 \mathrm{E}+06$ & 0.997 \\
\hline All Liquids & $1.10 \mathrm{E}+04$ & $1.17 \mathrm{E}+04$ & 0.936 \\
\hline All Solids & $1.86 \mathrm{E}+06$ & $1.87 \mathrm{E}+06$ & 0.995 \\
\hline
\end{tabular}

These calculations were done with a spreadsheet that carries more significant figures than displayed in Table 1. The fraction data given in Column 4 may therefore not exactly reflect the ratio of the Column 2 and 3 values based on the number of significant figures displayed in Table 1. The fraction of the dose for the selected isotopes exceeds $95 \%$ for 
all but the SST liquids and All Liquids composites. The totals for these two composites are around $93 \%$. The ${ }^{241} \mathrm{Pu}$ contributes about $5.2 \%$ and the ${ }^{154} \mathrm{Eu}$ contributes about $1.4 \%$ to both the SST Liquids and the All Liquids composite. Both of these isotopes are beta emitters and are not included in the selected isotopes.

\subsection{Ingestion Doses}

Twenty-four hour ingestion doses were calculated with the GENII code (Napier, 1988) as described in WHC-SD-WM-SARR-016 (1996). The calculation of the fraction of the ingestion doses for the selected isotopes are shown in Appendix B and the results are summarized in Table 2.

Table 2. Fraction of ULD for Ingestion Doses for ${ }^{80} \mathrm{Sr},{ }^{90} Y_{1}{ }^{137} \mathrm{Cs}$, and the Alpha Emitters.

\begin{tabular}{|l|c|c|c|}
\hline Composite & $\begin{array}{c}\text { Ingestion ULD for } \\
\text { Selected lsotopes } \\
(\text { Sv-m }\end{array}$ & $\begin{array}{c}\text { Ingestion } / \mathrm{s}) \\
\text { ULD for all Isotopes } \\
\left(\text { Sv-m }{ }^{3} / \mathrm{L}-\mathrm{s}\right)\end{array}$ & $\begin{array}{c}\text { Fraction of } \\
\text { Contribution of } \\
\text { Selected Isotopes* }\end{array}$ \\
\hline SST Liquids & $5.25 \mathrm{E}-02$ & $5.33 \mathrm{E}-02$ & 0.985 \\
\hline SST Solids & $4.07 \mathrm{E}+00$ & $4.08 \mathrm{E}+00$ & 0.999 \\
\hline DST Liquids & $6.69 \mathrm{E}-02$ & $6.69 \mathrm{E}-02$ & 0.999 \\
\hline DST solids & $4.88 \mathrm{E}-01$ & $4.93 \mathrm{E}-01$ & 0.990 \\
\hline AWF Liquids & $9.13 \mathrm{E}-02$ & $9.13 \mathrm{E}-02$ & 1.000 \\
\hline AWF Solids & $8.06 \mathrm{E}+00$ & $8.07 \mathrm{E}+00$ & 0.999 \\
\hline All Liquids & $1.11 \mathrm{E}-01$ & $1.11 \mathrm{E}-01$ & 0.993 \\
\hline All Solids & $8.15 \mathrm{E}+00$ & $8.16 \mathrm{E}+00$ & 0.999 \\
\hline
\end{tabular}

- See Appendix $\mathbf{B}$ for detailed calculation

The fractions were determined with a spreadsheet that carries more significant figures than displayed in Table 2. The data given in Column 4 may therefore not exactly reflect the ratio of the column 2 and 3 values calculated based on the number of significant figures given in the table. Since GENII displays dose calculation results to only two significant figures, the fractions may not be exact. However, the data does support the conclusion that considering only ${ }^{90} \mathrm{Sr},{ }^{80} \mathrm{Y},{ }^{137} \mathrm{Cs}$, and the alpha emitters includes well over $95 \%$ of the dose.

\subsection{Effective Dose Conversion Factor for Alpha Emitters}

Sample data from the tanks frequently give total alpha activity rather than activity from specitic isotopes. A calculation of effective dose conversion factors for all the alpha emitting isotopes has been made. This dose conversion factor could therefore be used with the total alpha activity in a similar manner as the dose conversion factors for specific activity. Effective dose conversion factors for the alpha emitters for each composite are calculated using the following formula:

$$
D C F_{\text {oft }}=\frac{\Sigma\left(D C F_{1}\right)\left(A_{1}\right)}{\Sigma\left(A_{1}\right)}
$$

Where $D_{C F}=$ Effective dose conversion factor

$D_{C F}=$ Dose conversion factors for the ith isotope

$A_{1}=$ Activity of the ith isotope 
The alpha emitting isotopes are ${ }^{237} \mathrm{~Np},{ }^{238} \mathrm{Pu},{ }^{230} \mathrm{Pu},{ }^{241} \mathrm{Am}$, and ${ }^{24} \mathrm{Cm}$. The calculations are shown in Appendix $C$ and the results are summarized in Table 3.

Table 3. Effective Dose Conversion Factor for Alpha Emitters for Different Composites

\begin{tabular}{|l|c|}
\hline Composite & $\begin{array}{l}\text { Effective Dose Conversion } \\
\text { Factor for Tank Waste } \\
\text { Alpha Emitters (Sv/Bq) }\end{array}$ \\
\hline SST Liquids & 1.17 E-04 \\
\hline SST Solids & 1.16 E-04 \\
\hline DST Liquids & 1.19 E-04 \\
\hline DST solids & 1.18 E-04 \\
\hline AWF Liquids & 1.19 E-04 \\
\hline AWF Solids & 1.22 E-04 \\
\hline All Liquids & 1.18 E-04 \\
\hline All Solids & 1.21 E-04 \\
\hline
\end{tabular}

An effective dose conversion factor for alpha emitters for ingestion doses was not calculated since the alpha emitters are a small fraction of the ingestion dose, and the ingestion dose is a relative small fraction of the total doses.

\subsection{References}

EPA, 1988, Limiting Values of Radionuclide Intake and Air Concentrations and Dose Conversion Factors for Inhalation, Submersion and Ingestion, Federal Guidance Report Number 11, Oak Ridge National Laboratory.

Napier, B. A., 1988, GENII - the Hanford Environmental Radiation Dosimetry Software System, PNL 6584, Richland, WA.

WHC-SD-WM-SARR-016, 1996, Tank Waste Compositions and Atmospheric Dispersion Coefificients for Use in Safety Analysis Consequence Assessments, Rev. 2, Westinghouse Hanford Company, Richland, Washington 
HNF- 4754, Rev 0

Appendix A Unit Liter Dose Calculation 
The spreadsheats for the calculations of the ULD and the fractional contribution from

${ }^{\infty} \mathrm{Sr},{ }^{\infty} Y,{ }^{137} \mathrm{Cs}$ and alpha emitters are given in this Appendix. See WHC-SD-WM-SARR-

016 for a complete explanation of the ULD calculation procedure.

Table A-1. Unit Liter Doses and ${ }^{80} \mathrm{Sr},{ }^{20},{ }^{137} \mathrm{Cs}$ and Alpha Emitters Fractional Contribution for SST Liquids and Solids

\begin{tabular}{|c|c|c|c|c|c|}
\hline \multirow[b]{2}{*}{ ISOTOPE } & \multirow[b]{2}{*}{ DCF $(\mathrm{Sv} / \mathrm{Bq})$} & \multicolumn{2}{|c|}{ SST LIQUIDS } & \multicolumn{2}{|c|}{ SST SOLIDS } \\
\hline & & $\overline{B q} / \mathbf{L}$ & ULD Sv/L & $\overline{B q / L}$ & $\overline{S v / L}$ \\
\hline$\overline{C-14}$ & $5.64 E-10$ & $1.00 E+05$ & 5.64E-05 & $1.20 E+05$ & 6.77E-05 \\
\hline Co-60 & 5.91E-08 & $9.53 E+06$ & 5.63E-01 & $4.18 E+08$ & 2.47E+01 \\
\hline Se-79 & 2.63E-09 & $0.00 E+01$ & $0.00 E+01$ & $1.70 E+04$ & $4.52 E-05$ \\
\hline Sr-90 & 6.47E-08 & $1.05 E+10$ & $6.79 E+02$ & $1.63 E+12$ & $1.05 E+05$ \\
\hline$\overline{Y-90}$ & 2.28E-09 & $1.05 E+10$ & $2.39 E+01$ & $1.63 E+12$ & $3.72 E+03$ \\
\hline Tc-99 & 2.25E-09 & $1.70 E+07$ & $3.83 E-02$ & $1.20 E+10$ & $2.70 E+01$ \\
\hline$R u-103$ & 1.29E-07 & $9.93 E+02$ & $1.28 \mathrm{E}-04$ & $7.22 E+04$ & 9.31E-03 \\
\hline Sb-125 & 3.30E-09 & $3.42 E+04$ & 1.13E-04 & $1.81 E+08$ & 5.97E-01 \\
\hline $1-129$ & 4.69E-08 & $1.00 E+04$ & $4.69 \mathrm{E}-04$ & $6.40 E+06$ & 3.00E-01 \\
\hline Cs-134 & $1.25 E-08$ & 1.17E+05 & 1.46E-03 & $1.44 E+03$ & 1.80E-02 \\
\hline Cs-137 & $8.63 E-09$ & $2.21 E+10$ & 1.91E+02 & $1.01 E+11$ & $8.72 E+02$ \\
\hline Ce-144 & 1.01E-07 & 9.05E-00 & $9.14 E-07$ & $3.37 E+02$ & $3.40 E-05$ \\
\hline$P m-147$ & $1.06 E-08$ & $0.00 E+01$ & $0.00 E+01$ & $0.00 E+01$ & $0.00 E+01$ \\
\hline Eu-154 & $7.73 E-08$ & $2.35 E+09$ & $1.82 E+02$ & $5.75 E+09$ & $4.44 E+02$ \\
\hline Eu-155 & 1.12E-08 & $5.87 E+07$ & 6.57E-01 & $5.01 E+06$ & 5.61E-02 \\
\hline Np-237 & $1.46 E-04$ & $0.00 E+01$ & $0.00 E+01$ & $3.02 E+07$ & 4.41E+03 \\
\hline $\mathrm{Pu}-238$ & $1.06 E-04$ & $9.21 E+04$ & $9.76 \mathrm{E}-00$ & $1.87 E+08$ & $1.98 E+04$ \\
\hline Pu-239 & $1.16 E-04$ & $3.62 E+07$ & $4.20 E+03$ & $4.40 E+08$ & $5.10 E+04$ \\
\hline Pu-241 & 2.23E-03 & $2.57 E+08$ & $5.73 E+02$ & $3.22 E+09$ & $7.18 E+03$ \\
\hline Am-241 & $1.20 E-04$ & $4.23 E+07$ & $5.08 E+03$ & $2.29 E+08$ & $2.75 E+04$ \\
\hline $\mathrm{Cm}-242$ & 4.67E-06 & $0.00 E+01$ & $0.00 E+01$ & $0.00 E+01$ & $0.00 E+01$ \\
\hline $\mathrm{Cm}-244$ & $6.705-05$ & $4.23 E+05$ & $2.83 E+01$ & $2.29 E+03$ & $1.53 E+02$ \\
\hline Total & & & $1.10 E+04$ & & $2.21 E+05$ \\
\hline \multicolumn{3}{|c|}{ Contribution from Sr-90 + Y-90 +Cs-137 + alpha* } & $1.02 E+04$ & & $2.13 E+05$ \\
\hline \multicolumn{3}{|c|}{ Fraction from Sr-90 + Y-90 +Cs-137 + alpha* } & 9.31E-01 & & 9.65E-01 \\
\hline
\end{tabular}

- Alpha emitters are Np-237, Pu-238, Pu-239, Am-241, and Cm-244 
Table A-2. Unit Liter Doses and ${ }^{0} \mathrm{Sr},{ }^{20 Y},{ }^{137} \mathrm{Cs}$ and Alpha Emitters Fractional Contribution for DST Liquids and Solids

\begin{tabular}{|l|c|c|c|c|c|}
\hline \multirow{2}{*}{ ISOTOPE } & DCF (Sv/Bq) & \multicolumn{2}{|c|}{ DST LIQUIDS } & \multicolumn{2}{c|}{ DST SOLIDS } \\
\cline { 2 - 6 } & $5.64 E-10$ & $2.30 E+05$ & $1.30 E-04$ & $1.60 E+05$ & $9.02 E-05$ \\
\hline C-14 & $5.91 E-08$ & $6.97 E+06$ & $4.12 E-01$ & $1.45 E+07$ & $8.57 E-01$ \\
\hline Co-60 & $2.66 E-09$ & $0.00 E+01$ & $0.00 E+01$ & $0.00 E+01$ & $0.00 E+01$ \\
\hline Se-79 & $6.47 E-08$ & $4.59 E+09$ & $2.97 E+02$ & $5.16 E+10$ & $3.34 E+03$ \\
\hline Sr-90 & $2.28 E-09$ & $4.59 E+09$ & $1.05 E+01$ & $5.16 E+10$ & $1.18 E+02$ \\
\hline Y-90 & $2.25 E-09$ & $1.10 E+07$ & $2.48 E-02$ & $6.20 E+07$ & $1.40 E-01$ \\
\hline Rc-99 & $1.29 E-07$ & $0.00 E+01$ & $0.00 E+01$ & $0.00 E+01$ & $0.00 E+01$ \\
\hline Sb-125 & $3.30 E-09$ & $0.00 E+01$ & $0.00 E+01$ & $0.00 E+01$ & $0.00 E+01$ \\
\hline I-129 & $4.69 E-08$ & $2.00 E+04$ & $9.38 E-04$ & $2.00 E+04$ & $9.38 E-04$ \\
\hline Cs-134 & $1.25 E-08$ & $6.11 E+06$ & $7.64 E-02$ & $9.44 E+06$ & $1.18 E-01$ \\
\hline Cs-137 & $8.63 E-09$ & $5.86 E+10$ & $5.06 E+02$ & $5.86 E+10$ & $5.06 E+02$ \\
\hline Ce-144 & $1.01 E-07$ & $0.00 E+01$ & $0.00 E+01$ & $0.00 E+01$ & $0.00 E+01$ \\
\hline Pm-147 & $1.06 E-08$ & $3.60 E+07$ & $3.82 E-01$ & $0.00 E+01$ & $0.00 E+01$ \\
\hline Eu-154 & $7.73 E-08$ & $4.18 E+07$ & $3.23 E-00$ & $3.00 E+08$ & $2.32 E+01$ \\
\hline Eu-155 & $1.12 E-08$ & $0.00 E+01$ & $0.00 E+01$ & $0.00 E+01$ & $0.00 E+01$ \\
\hline Np-237 & $1.46 E-04$ & $2.30 E+05$ & $3.36 E+01$ & $8.11 E+05$ & $1.18 E+02$ \\
\hline Pu-238 & $1.06 E-04$ & $1.78 E+06$ & $1.89 E+02$ & $7.15 E+07$ & $7.58 E+03$ \\
\hline Pu-239 & $1.16 E-04$ & $7.65 E+06$ & $8.87 E+02$ & $1.57 E+09$ & $1.82 E+05$ \\
\hline Pu-241 & $2.23 E-06$ & $1.84 E+07$ & $4.10 E+01$ & $3.81 E+09$ & $8.50 E+03$ \\
\hline Am-241 & $1.20 E-04$ & $3.40 E+07$ & $4.08 E+03$ & $2.71 E+09$ & $3.25 E+05$ \\
\hline Cm-242 & $4.67 E-06$ & $1.13 E+02$ & $5.28 E-04$ & $0.00 E+01$ & $0.00 E+01$ \\
\hline Cm-244 & $6.70 E-05$ & $1.22 E+05$ & $8.17 E-00$ & $1.64 E+07$ & $1.10 E+03$ \\
\hline Total & & & $6.06 E+03$ & & $5.29 E+05$ \\
\hline Contribution from Sr-90+Y-90+Cs-137+alpha* & $6.01 E+03$ & & $5.20 E+05$ \\
\hline Fraction from Sr-90+Y-90+Cs-137+alpha* & $9.93 E-01$ & & $9.84 E-01$ \\
\hline & & & & & \\
\hline
\end{tabular}

"Alpha emitters are Np-237, Pu-238, Pu-239, Am-241, and Cm-244 
Table A-3. Unit Liter Doses and ${ }^{10} \mathrm{Sr},{ }^{0 \mathrm{Y}},{ }^{137} \mathrm{Cs}$ and Alpha Emitters Fractional Contribution for AWF Liquids and Solids

\begin{tabular}{|l|c|c|c|c|c|}
\hline \multirow{2}{*}{ ISOTOPE } & DCF (Sv/Bq) & \multicolumn{2}{|c|}{ AWF LIQUIDS } & \multicolumn{2}{c|}{ AWF SOLIDS } \\
\cline { 3 - 6 } & $5.64 E-10$ & Bq/L & Sv/L & BqL & Sv/L \\
\hline C-14 & $5.80 E+04$ & $3.27 E-05$ & $1.00 E+05$ & $5.64 E-05$ \\
\hline Co-60 & $5.91 E-08$ & $7.71 E+05$ & $4.56 E-02$ & $4.85 E+08$ & $2.87 E+01$ \\
\hline Se-79 & $2.66 E-09$ & $0.00 E+01$ & $0.00 E+01$ & $0.00 E+01$ & $0.00 E+01$ \\
\hline Sr-90 & $6.47 E-08$ & $5.60 E+09$ & $3.62 E+02$ & $2.88 E+12$ & $1.86 E+05$ \\
\hline Y-90 & $2.28 E-09$ & $5.60 E+09$ & $1.28 E+01$ & $2.88 E+12$ & $6.57 E+03$ \\
\hline Tc-99 & $2.25 E-09$ & $1.20 E+07$ & $2.70 E-02$ & $2.80 E+08$ & $6.30 E-01$ \\
\hline Ru-106 & $1.29 E-07$ & $0.00 E+01$ & $0.00 E+01$ & $0.00 E+01$ & $0.00 E+01$ \\
\hline Sb-125 & $3.30 E-09$ & $0.00 E+01$ & $0.00 E+01$ & $0.00 E+01$ & $0.00 E+01$ \\
\hline I-129 & $4.69 E-08$ & $4.40 E+01$ & $2.05 E-06$ & $4.10 E+03$ & $1.92 E-01$ \\
\hline Cs-134 & $1.25 E-08$ & $1.33 E+04$ & $1.66 E-04$ & $0.00 E+01$ & $0.00 E+01$ \\
\hline Cs-137 & $8.63 E-09$ & $8.84 E+10$ & $7.63 E+02$ & $9.80 E+10$ & $8.46 E+02$ \\
\hline Ce-144 & $1.01 E-07$ & $0.00 E+01$ & $0.00 E+01$ & $0.00 E+01$ & $0.00 E+01$ \\
\hline Pm-147 & $1.06 E-08$ & $0.00 E+01$ & $0.00 E+01$ & $0.00 E+01$ & $0.00 E+01$ \\
\hline Eu-154 & $7.73 E-08$ & $0.00 E+01$ & $0.00 E+01$ & $1.11 E+10$ & $8.58 E+02$ \\
\hline Eu-155 & $1.12 E-08$ & $0.00 E+01$ & $0.00 E+01$ & $0.00 E+01$ & $0.00 E+01$ \\
\hline Np-237 & $1.46 E-04$ & $9.20 E+04$ & $1.34 E+01$ & $9.92 E+08$ & $1.45 E+05$ \\
\hline Pu-238 & $1.06 E-04$ & $2.75 E+03$ & $2.92 E-01$ & $6.74 E+07$ & $7.14 E+03$ \\
\hline Pu-239 & $1.16 E-04$ & $1.20 E+06$ & $1.39 E+02$ & $4.40 E+08$ & $5.10 E+04$ \\
\hline Pu-241 & $2.23 E-06$ & $3.39 E+05$ & $7.56 E-01$ & $1.65 E+09$ & $3.68 E+03$ \\
\hline Am-241 & $1.20 E-04$ & $1.10 E+06$ & $1.32 E+02$ & $1.10 E+10$ & $1.32 E+06$ \\
\hline Cm-242 & $4.67 E-06$ & $0.00 E+01$ & $0.00 E+01$ & $1.99 E+02$ & $9.29 E-04$ \\
\hline Cm-244 & $6.70 E-05$ & $1.10 E+04$ & $7.37 E-01$ & $6.08 E+07$ & $4.07 E+03$ \\
\hline Total & & & $1.42 E+03$ & & $1.73 E+06$ \\
\hline Contribution from Sr-90+Y90+Cs137+alpha* & $1.42 E+03$ & & $1.72 E+06$ \\
\hline Fraction from Sr-90+Y90+Cs137+alpha* & $9.99 E-01$ & & $9.97 E-01$ \\
\hline & & & & & \\
\hline
\end{tabular}

Alpha emitters are Np-237, Pu-238, Pu-239, Am-241, and Cm-244 
Table A-4. Unit Liter Doses and ${ }^{\infty 0} \mathrm{Sr},{ }^{\infty} \mathrm{Y},{ }^{137} \mathrm{Cs}$ and Alpha Emitters Fractional Contribution for All Liquids and All Solids

\begin{tabular}{|c|c|c|c|c|c|}
\hline \multirow[b]{2}{*}{ ISOTOPE } & \multirow[b]{2}{*}{ DCF (Sv/Eq) } & \multicolumn{2}{|c|}{$\begin{array}{r}\text { ALL LIQUIDS } \\
\end{array}$} & \multicolumn{2}{|c|}{ ALL SOLIDS } \\
\hline & & $B q / L$ & Sv/L & $B q \Omega$ & Sv/L \\
\hline C-14 & $5.64 E-10$ & $2.30 E+05$ & $1.30 E-04$ & $1.60 E+05$ & $9.025-05$ \\
\hline Co-60 & $5.91 E-08$ & $9.53 E+06$ & $\overline{5.63 E-01}$ & $4.85 E+08$ & 2.87E+01 \\
\hline Se-79 & $2.665-09$ & $0.00 E+01$ & $0.00 E+01$ & $1.70 E+04$ & $4.52 E-05$ \\
\hline Sr-90 & 6.47E-08 & $1.05 E+10$ & $6.79 E+02$ & $2.88 E+12$ & $1.86 E+05$ \\
\hline$Y-90$ & $2.28 E-09$ & $1.05 E+10$ & $2.39 E+01$ & $2.88 E+12$ & $6.57 E+03$ \\
\hline TC-99 & 2.25E-09 & $1.70 E+07$ & $3.83 E-02$ & $1.20 E+10$ & $2.70 E+01$ \\
\hline Ru-103 & 1.29E-07 & $9.93 E+02$ & $1.28 E-04$ & $7.22 E+04$ & $9.31 E-03$ \\
\hline$S b-125$ & 3.30E-09 & $3.42 E+04$ & $1.13 E-04$ & $1.81 E+08$ & 5.97E-01 \\
\hline $1-129$ & 4.69E-08 & $2.00 E+04$ & 9.38E-04 & $6.40 E+06$ & 3.00E-01 \\
\hline Cs-134 & 1.25E-08 & $6.11 E+03$ & $7.64 E-02$ & $9.44 E+06$ & 1.18E-01 \\
\hline Cs-137 & 8.63E-09 & $8.84 E+10$ & $7.63 E+02$ & $1.01 E+11$ & $8.72 E+02$ \\
\hline$C e-144$ & 1.01E-07 & $9.05 E-00$ & $9.14 E-07$ & $3.37 E+02$ & $3.40 E-05$ \\
\hline$P m-147$ & 1.06E-08 & $3.60 E+07$ & 3.82E-01 & $0.00 E+01$ & $0.00 E+01$ \\
\hline Eu-154 & $7.73 E-08$ & $2.35 E+09$ & $1.82 E+02$ & $1.11 E+10$ & $8.58 E+02$ \\
\hline Eu-155 & $1.125-08$ & $5.87 E+07$ & 6.57E-01 & $5.01 E+03$ & $5.61 E-02$ \\
\hline Np-237 & $1.46 E-04$ & $2.30 E+05$ & $3.36 E+01$ & $9.92 E+08$ & $1.45 E+05$ \\
\hline$P u-238$ & $1.00 \mathrm{E}-04$ & $1.78 E+06$ & $1.89 E+02$ & $1.87 E+08$ & $1.98 E+04$ \\
\hline Pu-239 & $1.16 E-04$ & $3.62 E+07$ & $4.20 E+03$ & 1.57E+09 & $1.82 E+05$ \\
\hline Pu-241 & 2.23E-06 & $2.57 E+08$ & $5.73 E+02$ & $3.81 E+09$ & $8.50 E+03$ \\
\hline Am-241 & $1.20 E-04$ & $4.23 E+07$ & $5.08 E+03$ & $1.10 E+10$ & $1.32 E+06$ \\
\hline $\mathrm{Cm}-242$ & 4.67E-06 & $1.13 E+02$ & $5.28 E-04$ & $1.99 E+02$ & $9.29 E-04$ \\
\hline $\mathrm{Cm}-244$ & $6.70 E-05$ & $4.23 E+05$ & $2.83 E+01$ & $6.08 E+07$ & $4.07 E+03$ \\
\hline Total & & & 1.17E+04 & & 1.87E+06 \\
\hline \multicolumn{3}{|c|}{ Contribution from Sr-90+ Y90+ Cs137 + alpha* } & $1.10 E+04$ & & $1.86 \mathrm{E}+06$ \\
\hline \multicolumn{3}{|c|}{ Fraction from Sr-90 + Y90 +Cs137 + alpha } & 9.36E-01 & & 9.95E-01 \\
\hline
\end{tabular}

Alpha emitters are Np-237, Pu-238, Pu-239, Am-241, and Cm-244 
HNF- 4754, Rev 0

Appendix B Detailed Calculations for Ingestion Dose Fractions 
Ingestion doses for individual isotopes are taken from the GENII output files for the ingestion ULD calculation given in SARR-016 (1996). The first group includes the selected isotopes (Sr-90 + Y-90 + Cs-137 + the alpha emitters). Isotopes listed under "Other" are the isotopes not included in the list. The original calculation included the 11 tracked isotopes plus the 11 additional isotopes. Only the eleven tracked isotopes are included in this calculation since the contribution for the other isotopes are negligible. (See WHC-SD-WM-SARR-016, 1996). The fraction is the total of the doses from the eight selected isotopes divided by the dose from all eleven isotopes. The results are presented in Tables B1 and B-2.

Table B-1. Ingestion Dose Calculation for SST and DST Solids and Liquids

\begin{tabular}{|c|c|c|c|c|}
\hline Isotope & $\begin{array}{l}\text { Ingestion Dose } \\
\text { Contribution for } \\
\text { Each lsotope for } \\
\text { SST Liquids (Sv) }\end{array}$ & $\begin{array}{l}\text { Ingestion Dose } \\
\text { Contribution for } \\
\text { Each Isotope for } \\
\text { SST Solids (SV) }\end{array}$ & $\begin{array}{l}\text { Ingestion Dose } \\
\text { Contribution for } \\
\text { Each Isotope for } \\
\text { DST Liquids (Sv) }\end{array}$ & $\begin{array}{l}\text { Ingestion Dose } \\
\text { Contribution for } \\
\text { Each Isotope for } \\
\text { DST Solids (Sv) }\end{array}$ \\
\hline Sr-90 & $2.50 E-02$ & 3.60E-00 & $1.00 E-02$ & 1.20E-01 \\
\hline$Y-90$ & $2.20 E-03$ & 3.20E-01 & $9.10 E-04$ & 1.00E-02 \\
\hline Cs-137 & 2.00E-02 & 9.00E-02 & 5.30E-02 & $5.30 E-02$ \\
\hline$N p-237$ & $0.00 E+00$ & 3.20E-03 & 2.30E-05 & 8.10E-05 \\
\hline Pu-238 & 5.60E-06 & $1.20 \mathrm{E}-02$ & 1.10E-04 & $4.40 E-03$ \\
\hline Pu-239 & $2.40 E-03$ & $2.90 E-02$ & $5.20 E-04$ & $1.10 E-01$ \\
\hline Am-241 & 2.90E-03 & 1.60E-02 & 2.30E-03 & 1.90E-01 \\
\hline $\mathrm{Cm}-244$ & 1.60E-05 & $8.60 E-05$ & 4.50E-06 & $6.00 E-04$ \\
\hline Total & 5.25E-02 & 4.07E-00 & 6.69E-02 & 4.88E-01 \\
\hline \multicolumn{5}{|l|}{ Other lsotopes } \\
\hline Co-60 & 4.60E-06 & 2.00E-04 & 3.40E-06 & $7.20 E-00$ \\
\hline Eu-154 & 4.40E-04 & 1.00E-03 & $7.50 E-06$ & $5.50 E-05$ \\
\hline Pu-241 & 3.30E-04 & 4.10E-03 & $2.30 E-05$ & 4.80E-03 \\
\hline Total all & 5.33E-02 & $4.08 E-00$ & 6.69E-02 & 4.93E-01 \\
\hline $\begin{array}{l}\text { Fraction } \\
\text { Selected/total }\end{array}$ & 9.85E-01 & 9.99E-01 & 9.99E-01 & 9.90E-01 \\
\hline
\end{tabular}


Table B-2. Ingestion Dose Calculation for AWF and All Solids and Liquids

\begin{tabular}{|c|c|c|c|c|}
\hline Isotope & $\begin{array}{l}\text { Ingestion dose } \\
\text { Contribution for } \\
\text { Each Isotope for } \\
\text { AWF Liquids (Sv) }\end{array}$ & $\begin{array}{l}\text { Ingestion dose } \\
\text { Contribution for } \\
\text { Each Isotope for } \\
\text { AWF Solids (Sv) }\end{array}$ & $\begin{array}{l}\text { Ingestion dose } \\
\text { Contribution for } \\
\text { Each Isotope for All } \\
\text { Liquids (Sv) }\end{array}$ & $\begin{array}{l}\text { Ingestion dose } \\
\text { Contribution for } \\
\text { Each Isotope for All } \\
\text { Solids (Sv) }\end{array}$ \\
\hline Sr-90 & $1.20 E-02$ & 6.50E-00 & 2.50E-02 & $6.50 E-00$ \\
\hline$Y-90$ & $1.10 E-03$ & 5.80E-01 & $2.20 E-03$ & 5.80E-01 \\
\hline Cs-137 & $7.80 E-02$ & 8.70 E-02 & $7.80 E-02$ & $8.70 E-02$ \\
\hline Np-237 & $9.20 E-03$ & $9.90 \mathrm{E}-02$ & 2.30E-05 & 9.90E-02 \\
\hline Pu-238 & 1.70E-07 & 4.10E-03 & $1.10 E-04$ & 1.20E-02 \\
\hline Pu-239 & $8.10=-05$ & $2.90 E-02$ & $2.40 E-03$ & 1.10E-01 \\
\hline Am-241 & $7.60 E-05$ & 7.60E-01 & 2.90E-03 & $7.605-01$ \\
\hline Cm-244 & $4.10 \sum-07$ & 2.30E-03 & $1.605-05$ & 2.30E-03 \\
\hline Total & $9.13 E-02$ & $8.06 \mathrm{E}-00$ & 1.11E-01 & $8.15 E-00$ \\
\hline \multicolumn{5}{|l|}{ Other Isotopes } \\
\hline Co-60 & 3.70E-07 & $2.40 E-04$ & 4.60E-03 & $2.40 E-04$ \\
\hline Eu-154 & $0.00 E+01$ & $2.00 E-03$ & $4.40 E-04$ & $2.00 E-03$ \\
\hline Pu-241 & 4.30E-07 & 2.10E-03 & 3.30E-04 & $4.80=-03$ \\
\hline Total & $9.13 E-02$ & 8.07E-00 & 1.11E-01 & $8.16 E-00$ \\
\hline $\begin{array}{l}\text { Fraction } \\
\text { Selected/total }\end{array}$ & $1.00 E+00$ & 9.99E-01 & $9.93 \mathrm{E}-01$ & $9.99 \mathrm{E}-01$ \\
\hline
\end{tabular}


HNF- 4754 , Rev 0

Appendix C Effective Dose Conversion Factor for the Alpha Emitters. 
The ULD contribution for each isotope is calculated by multiplying the activities by the dose conversion factor (DCF) in Tables C-1 through C-8. The ULD contribution for each isotope is calculated in Column 4 by multiplying the activity in Column 3 by the dose conversion factor in Column 2. The total ULD for all the alpha emitters is calculated by summing the ULDs for each isotope in Column 4. The total alpha activity is calculated by summing the activities in Column 2 . The effective DCF is the ULD divided by the total activity (Total of Column 4 divided by the total of Column 2).

Table C-1. Effective Dose Conversion Factor for Tank Farm Alpha Emitters-SST Liquids

\begin{tabular}{|c|c|c|c|}
\hline Isotope & $\begin{array}{r}\text { Activity/L } \\
\text { (Bg/L) }\end{array}$ & $\begin{array}{l}\text { DCF" } \\
\text { (Sv/Bq) }\end{array}$ & $\begin{array}{r}\text { DCF"activity } \\
\text { (Sv/L) }\end{array}$ \\
\hline Np-237 & $0.00 E+01$ & $1.46 E-04$ & $0.00 E+01$ \\
\hline$P_{1-238}$ & $9.20 E+04$ & $1.06 E-04$ & $9.75 E-00$ \\
\hline Pv-239 & $3.60 E+07$ & $1.16=-04$ & $4.18 E+03$ \\
\hline Am-241 & $4.20 E+07$ & $1.20=-04$ & $5.04 E+03$ \\
\hline $\mathrm{Cm}-244$ & $4.20 E+05$ & $6.70 E-05$ & $2.81 E+01$ \\
\hline Total & $7.90=+07$ & & $9.25 E+03$ \\
\hline \multicolumn{3}{|c|}{ Effective DCF (Sv/Ba) } & 1.17E-04 \\
\hline
\end{tabular}

Table C-2. Effective Dose Conversion Factor for Tank Farm Alpha Emitters-SST Solids

\begin{tabular}{|l|r|r|r|}
\hline Isotope & $\begin{array}{r}\text { Activity/L } \\
(\mathrm{Bg} / \mathrm{L})\end{array}$ & $\begin{array}{r}\text { DCF } \\
(\mathrm{Sv} / \mathrm{Bg})\end{array}$ & $\begin{array}{r}\text { DCF"activity } \\
\text { (SvL) }\end{array}$ \\
\hline Np-237 & $3.00 E+07$ & $1.46 E-04$ & $4.38 E+03$ \\
\hline Pu-238 & $1.00 E+08$ & $1.06 E-04$ & $2.01 E+04$ \\
\hline Pu-239 & $4.40 E+08$ & $1.16 E-04$ & $5.10 E+04$ \\
\hline Am-241 & $2.30 E+08$ & $1.20 E-04$ & $2.76 E+04$ \\
\hline Cm-244 & $2.30 E+06$ & $6.70 E-05$ & $1.54 E+02$ \\
\hline Total & $8.92 E+08$ & & $1.03 E+05$ \\
\hline Efiective DCF (Sv/Ba) & & $1.16 E-04$ \\
\hline
\end{tabular}

Table C-3. Effective Dose Conversion Factor for Tank Farm Alpha Emitters-DST Liquids

\begin{tabular}{|c|c|c|c|}
\hline Isotope & $\begin{array}{r}\text { Activity/L } \\
(\mathrm{Bg} L)\end{array}$ & $\begin{array}{l}\text { DCF" } \\
(\mathrm{SV} / \mathrm{Bg})\end{array}$ & $\begin{array}{r}\text { DCF"activity } \\
\text { (SvL) }\end{array}$ \\
\hline $\mathrm{Np}-237$ & $2.30=+05$ & $1.465-04$ & $3.36 E+01$ \\
\hline$P u-238$ & $1.80 E+06$ & $1.06=-04$ & 1.91E+02 \\
\hline Pu-239 & $7.70 E+06$ & $1.16=-04$ & $8.93 E+02$ \\
\hline Am-241 & $3.40 E+07$ & $1.20 E-04$ & $4.08 E+03$ \\
\hline $\mathrm{Cm}-244$ & $1.20 E+05$ & $6.70 E-05$ & $8.04 E-00$ \\
\hline Total & $4.39 E+07$ & & 5.21E+03 \\
\hline \multicolumn{3}{|c|}{ Effective DCF (SviBa) } & 1.19E-04 \\
\hline
\end{tabular}


Table C-4. Effective Dose Conversion Factor for Tank Farm Alpha Emitters-DST Solids

\begin{tabular}{|l|r|r|r|}
\hline Isotope & $\begin{array}{r}\text { Activity/L } \\
(\mathrm{EqL})\end{array}$ & $\begin{array}{r}\text { DCF" } \\
(\mathrm{Sv} / \mathrm{Bq})\end{array}$ & $\begin{array}{r}\text { DCF"activity } \\
(\mathrm{SvL})\end{array}$ \\
\hline Np-237 & $8.10 E+05$ & $1.46 E-04$ & $1.18 E+02$ \\
\hline Pu-238 & $7.20 E+07$ & $1.06 E-04$ & $7.63 E+03$ \\
\hline Pu-239 & $1.60 E+09$ & $1.16 E-04$ & $1.86 E+05$ \\
\hline Am-241 & $2.70 E+09$ & $1.20 E-04$ & $3.24 E+05$ \\
\hline Cm-244 & $1.60 E+07$ & $6.70 E-05$ & $1.07 E+03$ \\
\hline Total & $4.39 E+09$ & & $5.18 E+05$ \\
\hline Effective DCF (Sv/Bq) & & $1.18 E-04$ \\
\hline
\end{tabular}

Table C-5. Effective Dose Conversion Factor for Tank Farm Alpha Emitters-AWF Liquids

\begin{tabular}{|c|c|c|c|}
\hline Isotope & $\begin{array}{r}\text { Activity/L } \\
\text { (Eq) }\end{array}$ & $\begin{array}{r}\mathrm{DCF} \\
(\mathrm{Sv} / \mathrm{Bg})\end{array}$ & $\begin{array}{r}\text { DCF"activity } \\
\text { (Sv/L) }\end{array}$ \\
\hline No-237 & $9.20 E+04$ & $1.46 E-04$ & $1.34 E+01$ \\
\hline Pu-238 & $2.80 E+03$ & 1.06E-04 & 2.97E-01 \\
\hline Pu-239 & $1.20 E+06$ & 1.16E-04 & $1.39 E+02$ \\
\hline Am-241 & $1.10 E+06$ & $1.20 E-04$ & $1.32 E+02$ \\
\hline Cm-244 & $1.10 E+04$ & $6.70 E-05$ & 7.37E-01 \\
\hline Total & $2.41 E+06$ & & $2.86 E+02$ \\
\hline Effective & & & $1.19 E-04$ \\
\hline
\end{tabular}

Table C-6. Effective Dose Conversion Factor for Tank Farm Alpha Emitters-AWF Solids

\begin{tabular}{|l|r|r|r|}
\hline Isotope & $\begin{array}{r}\text { Activity/L } \\
(\mathrm{Eq} / \mathrm{L})\end{array}$ & $\begin{array}{r}\text { DCF" } \\
(\mathrm{Sv} / \mathrm{Bq})\end{array}$ & $\begin{array}{r}\text { DCF"activity } \\
(\mathrm{Sv} / \mathrm{L})\end{array}$ \\
\hline $\mathrm{Np}-237$ & $9.90 E+08$ & $1.46 E-04$ & $1.45 E+05$ \\
\hline Pu-238 & $6.70 E+07$ & $1.03 E-04$ & $7.10 E+03$ \\
\hline Pu-239 & $4.40 E+08$ & $1.16 E-04$ & $5.10 E+04$ \\
\hline Am-241 & $1.10 E+10$ & $1.20 E-04$ & $1.32 E+06$ \\
\hline Cm-244 & $6.10 E+07$ & $6.70 E-05$ & $4.09 E+03$ \\
\hline Total & $1.26 E+10$ & & $1.53 E+06$ \\
\hline Effective DCF (Sv/Bg) & & $1.22 E-04$ \\
\hline
\end{tabular}


Table C-7. Effective Dose Conversion Factor for Tank Farm Alpha Emitters-All Liquids

\begin{tabular}{|c|c|c|c|}
\hline Isotope & $\begin{array}{r}\text { Activity/L } \\
(\mathrm{Eq} / L)\end{array}$ & $\begin{array}{l}\text { DCF" } \\
(S v / B q)\end{array}$ & $\begin{array}{r}\text { DCF“activity } \\
(\text { SvL })\end{array}$ \\
\hline Np-237 & $2.30 E+05$ & $1.46 E-04$ & $3.36 E+01$ \\
\hline Pu-238 & $1.80 E+06$ & $1.06 E-04$ & $1.91 E+02$ \\
\hline Pu-239 & $3.60 E+07$ & $1.16 E-04$ & $4.18 E+03$ \\
\hline Am-241 & $4.20 E+07$ & $1.20=-04$ & $5.04 E+03$ \\
\hline Cm-244 & $4.20 \mathrm{E}+05$ & 6.70E-05 & $2.81 E+01$ \\
\hline Total & $8.05 E+07$ & & $9.47 E+03$ \\
\hline
\end{tabular}

Table C-8. Effective Dose Conversion Factor for Tank Farm Alpha Emitters-All Solids

\begin{tabular}{|l|r|r|r|}
\hline Isotope & $\begin{array}{r}\text { Activity/L } \\
(\mathrm{Bg} / \mathrm{l})\end{array}$ & $\begin{array}{r}\text { DCF" } \\
(\mathrm{Sv} / \mathrm{Bq})\end{array}$ & $\begin{array}{r}\text { DCF*activity } \\
(\mathrm{Sv} / \mathrm{L})\end{array}$ \\
\hline Np-237 & $9.90 E+08$ & $1.46 E-04$ & $1.45 E+05$ \\
\hline Pu-238 & $1.90 E+08$ & $1.06 E-04$ & $2.01 E+04$ \\
\hline Pu-239 & $1.60 E+09$ & $1.16 E-04$ & $1.86 E+05$ \\
\hline Am-241 & $1.10 E+10$ & $1.20 E-04$ & $1.32 E+06$ \\
\hline Cm-244 & $6.10 E+07$ & $6.70 E-05$ & $4.09 E+03$ \\
\hline Total & $1.38 E+10$ & & $1.67 E+06$ \\
\hline Effective DCF (Sv/Ba) & & $1.21 E-04$ \\
\hline
\end{tabular}


HNF- 4754 , Rev 0

Appendix D Peer Review Checklist 


\section{TECHINICAL PEER REVIEWS}

\section{CHECKLIST FOR TECHNICAL PEER REVIEW}

Document Reviewed: HNF -4754 , ROV 0

Title: TaNk wiate Isotope rontribution

Author: J.C Van Keunen

Date: Junc 1999

Scope of Review: ENtine docunent

Yes No* NA

[ ] [ ] X ] ** Previous reviews complete and cover analysis, up to scope of this review, with no gaps.

$[X][][] \quad$ Problem completely defined.

[][]$[X]$

[X][ ] ]

$[x][][]$

$[X][][]$

$[X][][]$

Accident scenarios developed in a clear and logical manner.

Necessary assumptions explicitly stated and supported.

Computer codes and data files documented.

Data used in calculations explicitly stated in document.

$[X][][]$

Data checked for consistency with original source information as applicable.

$[X][][]$

Mathematical derivations checked including dimensional consistency of results.

Models appropriate and used within range of validity or use outside range of established validity justified.

[X] [ ] ] Hand calculations checked for errors. Spreadsheet results should be treated exactly the same as hand calculations.

[X] [ ] [ ] Software input correct and consistent with document reviewed.

$[X][][]$

$[\mathrm{l}][\mathrm{X}]$

Software output consistent with input and with results reported in document reviewed.

Limits/criteria/guidelines applied to analysis results are appropriate and referenced.

Limits/criteria/guidelines checked against references.

$[\mathrm{l}][\mathrm{X}]$

$[x][][]$

Safety margins consistent with good engineering practices.

$[X][$ ] ]

$[X][][]$

Conclusions consistent with analytical results and applicable limits.

[]$[X] * *$

Results and conclusions address all points required in the problem statement.

Format consistent with applicable guides or other standards.

$[X][][]$

Review calculations, comments, and/or notes are attached.

Document approved (for example, the reviewer affirms the technical accuracy of the document).
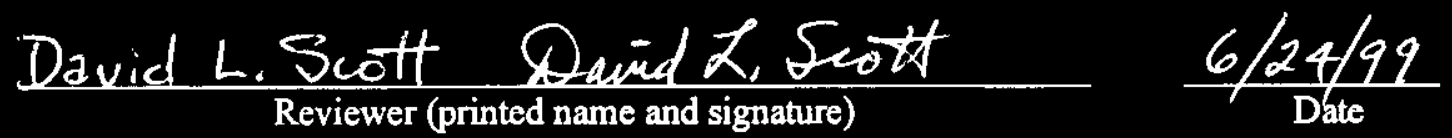

* All "no" responses must be explained below or on an additional sheet.

** Any calculations, comments, or notes generated as part of this review should be signed, dated, and attached to this checklist. Such material should be labeled and recorded in such a manner as to be intelligible to a technically qualified third party. 


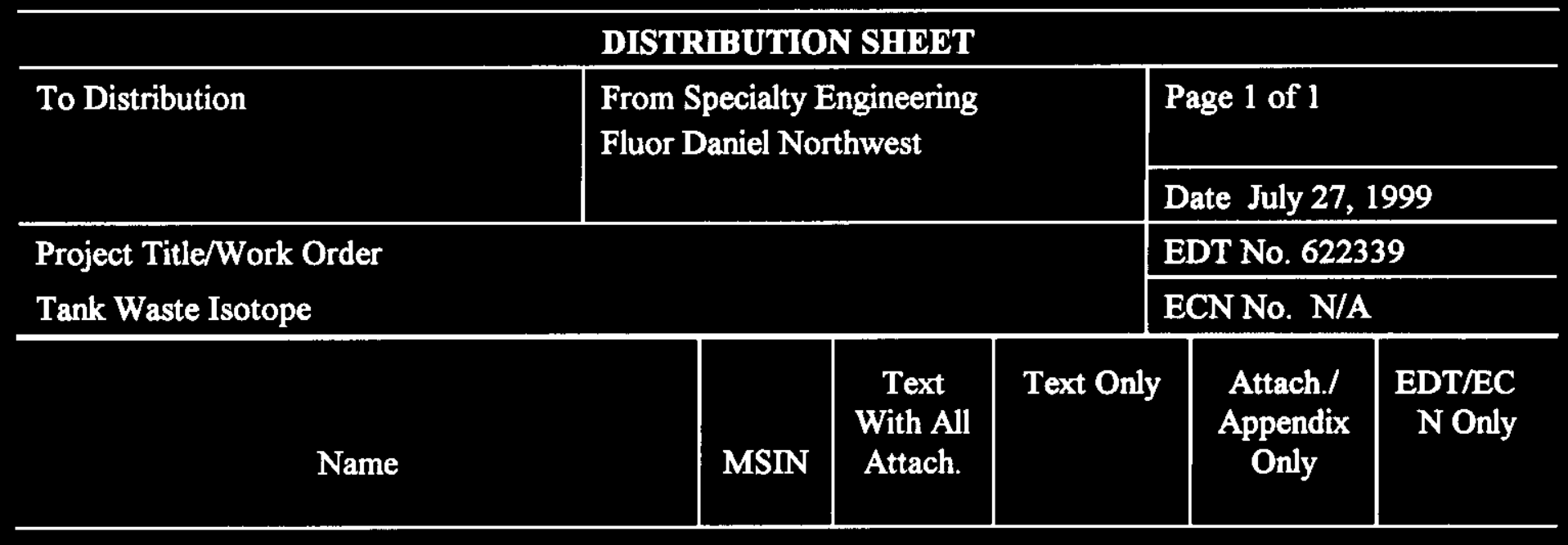

Lockheed Martin Hanford Company

W. L. Cowley

J. S. Davis

R1-49 $\quad X$
R1-49

D. A. Reynolds

R1-49 X

R2-11 X

Fluor Daniel Northwest, Inc.
B. E. Hey
J. C. Van Keuren
$\begin{array}{ll}B 4-47 & X \\ B 4-47 & X\end{array}$
Central Files (2)
B1-07 X
DOE/RL Reading Room
H2 -53
$\mathrm{X}$ 